\section{Jorge Sabato y el Pensamiento Latinoamericano en Ciencia, Tecnología, Desarrollo y Dependencia}

Resumen: En este artículo se analiza el rol de Jorge Sabato como una de las figuras clave del Pensamiento Latinoamericano en Ciencia, Tecnología, Desarrollo y Dependencia (PLACTED). Se muestra cómo a partir de sus trabajos en la industria metalúrgica consiguió crear, en la Comisión Nacional de Energía Atómica (CNEA) argentina, la base para los principales desarrollos autónomos del país en tecnología nuclear, en una síntesis de pensamiento y acción. Por otro lado, se describe cómo desde la práctica, desarrolló una línea de pensamiento en torno a la autonomía tecnológica, y cómo supo aglutinar, en base a su capacidad de liderazgo, a tecnólogos y pensadores de distintos países de la región que constituyeron el PLACTED. Se destacan sus trabajos en prospectiva y el carácter pionero a nivel mundial que significó el desarrollo de los conceptos de innovación, sistemas de innovación, la desagregación del paquete tecnológico, la tecnología como mercancía, y la difundida figura del llamado "Triángulo de Sabato".

Palabras clave: Sabato, Triángulo de Sabato, autonomía tecnológica, Pensamiento latinoamericano, Innovación, PLACTED.

Jorge Sabato and the Latin American Thought in Science, Technology, Development and Dependency

Summary: This article analyzes the role of Jorge Sabato as one of the key figures of Latin American Thought in Science, Technology, Development and Dependence (PLACTED). Based on his work in the metallurgical industry, it is shown how he managed to create the basis for the country's main autonomous developments in nuclear technology in the Argentine National Atomic Energy Commission (CNEA), in a combination of thought and action. On the other hand, it is described how he developed a line of thought around technological autonomy putting it into practice, and how he was able to bring together technologists and thinkers from different countries of the region that constituted PLACTED, based on his leadership capacity. His outstanding work in foresight and the pioneering nature at the world level meant the development of innovation concepts, innovation systems, the unbundling of the technological package, technology as a commodity, and the widespread figure of the so-called "Sabato Triangle".

Keywords: Sabato, Sabato Triangle, technological autonomy, Latin American thought, Innovation.

Jorge Sábato e o pensamento latino-americano em ciência, tecnologia, desenvolvimento e dependência

Resumo: Este artigo analisa o papel de Jorge Sabato como uma das figuras-chave do Pensamento Latino-Americano em Ciência, Tecnologia, Desenvolvimento e Dependência (PLACTED). Mostra-se como, a partir de seu trabalho na indústria metalúrgica, conseguiu criar na Comissão Nacional de Energia Atômica da Argentina (CNEA), a base para os principais desenvolvimentos autônomos do país em tecnologia nuclear, numa síntese de pensamento e ação. Por outro lado, descreve-se como, a partir da prática, desenvolveu uma linha de pensamento em torno da autonomia tecnológica, e como soube reunir, a partir de sua capacidade de liderança, tecnólogos e pensadores de diferentes países da região que se constituíram no PLACTED. Destacam-se seu trabalho de previsão e o pioneirismo em nível mundial que significou o desenvolvimento de conceitos de inovação, sistemas de inovação, a desagregação do pacote tecnológico, a tecnologia como mercadoria e a figura difundida do chamado "Triângulo de Sabato"

Palavras chave: Sabato, Triângulo de Sabato, autonomia tecnológica, pensamento latino-americano, Inovação

\section{Ciencia Tecnología \\ y Política}

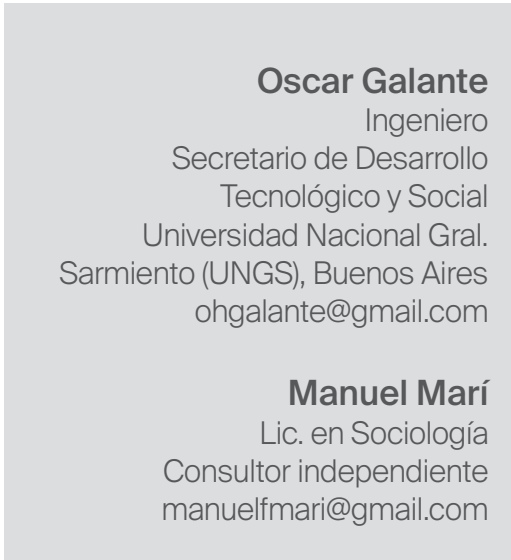

Año $3 \mathrm{~N}^{\circ} 5$ Noviembre 2020 Fecha de recibido: $26 / 08 / 2020$ Fecha de aprobado: 06/10/2020 https://doi.org/10.24215/26183188e048 https://revistas.unlp.edu.ar/CTyP ISSN 2618-3188

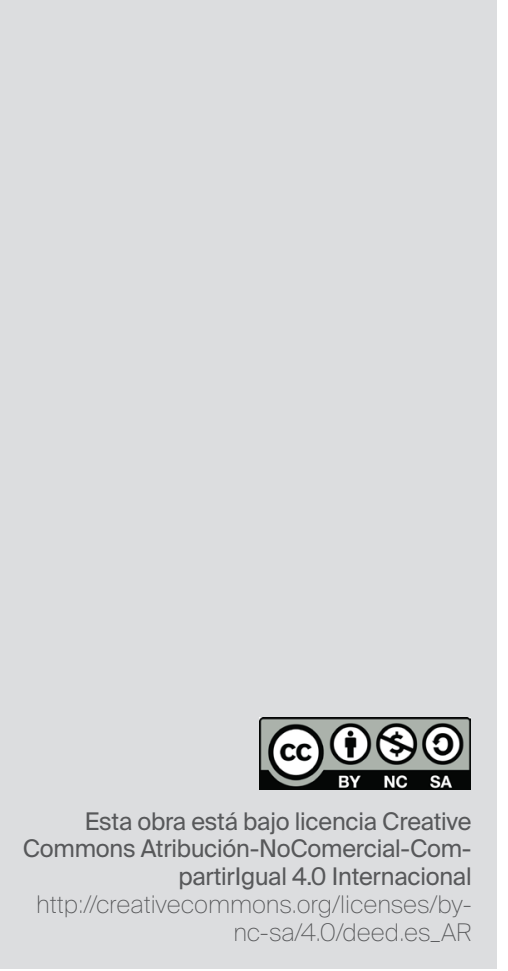


| Ciencia, Tecnología y Política | Año 3 | N5 | Noviembre 2020 | ISSN 2618-3188 | www.revistas.un|p.edu.ar/CTyP |

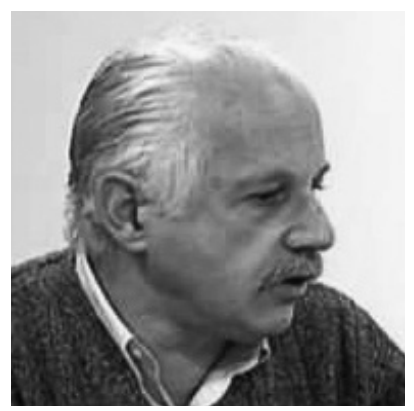

\section{Oscar Galante}

Ingeniero, Secretario de Desarrollo Tecnológico y Social Universidad Nacional Gral. Sarmiento (UNGS), Buenos Aires ohgalante@gmail.com

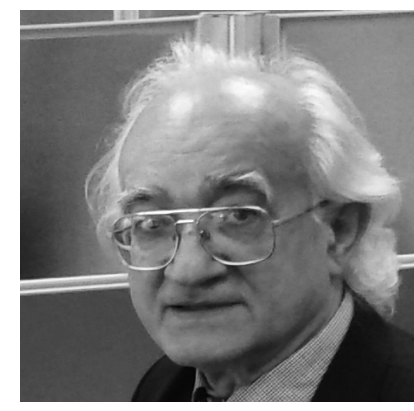

Manuel Marí

Lic. en Sociología

Consultor independiente

manuelfmari@gmail.com

\section{Jorge Sabato y el Pensamiento} Latinoamericano en Ciencia, Tecnología, Desarrollo y

\section{Dependencia}

Resumen: En este artículo se analiza el rol de Jorge Sabato como una de las figuras clave del Pensamiento Latinoamericano en Ciencia, Tecnología, Desarrollo y Dependencia (PLACTED). Se muestra cómo a partir de sus trabajos en la industria metalúrgica consiguió crear, en la Comisión Nacional de Energía Atómica (CNEA) argentina, la base para los principales desarrollos autónomos del país en tecnología nuclear, en una síntesis de pensamiento y acción. Por otro lado, se describe cómo desde la práctica, desarrolló una línea de pensamiento en torno a la autonomía tecnológica, y cómo supo aglutinar, en base a su capacidad de liderazgo, a tecnólogos y pensadores de distintos países de la región que constituyeron el PLACTED. Se destacan sus trabajos en prospectiva y el carácter pionero a nivel mundial que significó el desarrollo de los conceptos de innovación, sistemas de innovación, la desagregación del paquete tecnológico, la tecnología como mercancía, y la difundida figura del llamado "Triángulo de Sabato".

\section{Introducción}

Jorge Sabato se hizo conocido por la figura del "Triángulo de Sabato" (Sabato y Botana, 1968), que graficaba las interrelaciones que deberían existir entre el gobierno, la estructura productiva y la infraestructura científica y tecnológica, si se quería conseguir el objetivo de "lograr capacidad técnico-científica de decisión propia a través de la inserción de la Ciencia y la Técnica en la trama misma del proceso de desarrollo" (Sabato, 2011: 216). Pero ésta fue la culminación de un doble recorrido que lo tuvo como protagonista principal. Por un lado, el desarrollo de tecnologías (innovaciones, diríamos hoy, término que además él introdujo como pionero en la materia), en la Comisión Nacional de Energía Atómica (CNEA) de Argentina, en la cual contribuyó en forma determinante a infundir la idea de la autonomía tecnológica nacional, que desde entonces la caracterizó. Al mismo tiempo, Sabato fue articulador importantísimo y fundamental del movimiento llamado Pensamiento Latinoamericano en Ciencia, Tecnología, Desarrollo y Dependencia (PLACTED). Este movimiento 
aglutinó a emprendedores tecnológicos, científicos e intelectuales que abogaban por una capacidad autónoma de decisión en materia de selección y desarrollo de tecnologías: un movimiento, en palabras del mismo Sabato, caracterizado por "la estrecha relación que ha habido entre pensamiento y acción" (Sabato, 2011: 485).

En este artículo se tratarán los siguientes aspectos:

Por un lado, el camino realizado por Sabato de la física a la metalurgia, y de la práctica a la excelencia científica. Se muestra cómo a partir de sus trabajos en la industria metalúrgica, consiguió crear la base para los principales desarrollos del país en tecnología nuclear. En segundo lugar, se muestra cómo Sabato desarrolló, a partir de la práctica, una línea de pensamiento en torno a la autonomía tecnológica, y cómo supo aglutinar, en base a una gran capacidad de liderazgo, a tecnólogos y pensadores de distintos países de la región para formar un grupo heterogéneo pero coherente que se dio en llamar PLACTED. Finalmente, se destaca su carácter pionero en temas como la prospectiva y la introducción de los conceptos de innovación y de sistemas de innovación, adelantándose a la incorporación de dicho concepto en las escuelas económicas.

\section{El rol de Sabato en el desarrollo de la me- talurgia en Argentina y la CNEA}

En un homenaje que se le realizó a Jorge Sabato a mediados de los 90, Carlos Martínez Vidal, gran amigo y continuador de su obra en la CNEA, decía de él:

Fue un autodidacta y una rara mezcla entre intuitivo y rigurosamente científico. Profesor de física, se fue formando en física moderna, a través del estudio (era un lector incansable) y la enseñanza y la amistad de personalidades notables a los que consultaba y de quienes apren- día, como Gregorio Klimovsky, Jorge Bosch, Alberto Maiztegui (con quien escribió dos libros de Física) y otros (Ciapuscio, 1994: 80).

Sin embargo, pronto debió abandonar su primera vocación académica y en 1952 (con 26 años de edad) se hizo cargo de la Dirección del Laboratorio de Investigaciones de la empresa metalúrgica Decker S.A., pionera en integrar la investigación a su actividad productiva. En 1954, se retiró de Decker para crear con el Dr. Luis Boschi una nueva empresa, IMET S.A., dedicada al apoyo de la industria metalúrgica y metal-mecánica argentina, la que "fue contratada por la CNEA para que la asesorara en el campo metalúrgico y de elementos combustibles. Allí repitió la experiencia de 'aprender haciendo' con igual éxito pero en mayor escala, demostrando además su notable capacidad gerencial" (Ciapuscio, 1994: 83). Es así como recaló en la CNEA en 1955, donde fue nombrado director del nuevo Departamento de Metalurgia, lugar en el que pudo combinar la pasión por la excelencia científica y la capacidad de inventiva. En la CNEA, Sabato impulsó lo que según él fue tal vez "el paso fundamental para la creación de una capacidad tecnológica propia... cuando en 1957 se decidió construir un reactor experimental, en lugar de comprarlo, como estaban haciendo muchos países del Tercer Mundo " (Martínez Vidal y Marí, 2002: 6; Galante, Benso, Carnota, Marí y Vasen, 2009: 4). Este fue el comienzo del PLACTED o, como gustaba decir Sabato: la Escuela de Pensamiento Latinoamericano en Ciencia, Tecnología y Desarrollo.

A esto siguió la construcción misma del primer reactor generador de energía, la Central Nuclear de Atucha, entre 1965-1974. Para su construcción, encomendada a la empresa Siemens, se comenzó a utilizar el concepto de desagregación del paquete tecnológico, una de las ideas centrales de la Escuela. Con esto se consiguió 
elevar el porcentaje del componente nacional en la construcción, del 33\% originalmente planeado, al 42\% (en la segunda central nuclear se elevó este porcentaje a 50\%). Además, el combustible nuclear [elemento básico de una central nuclear] fue desarrollado en la CNEA, justamente en el Departamento de Metalurgia. Otro aspecto central de la estrategia de la CNEA fue el estímulo a la industria metalúrgica nacional para la fabricación de componentes a través del SATI (Servicio de Asistencia Tecnológica a la Industria) [creado precisamente por Sabato] (Galante et al., 2009: 4).

Él mismo fue también uno de los que sugirieron posteriormente la creación del Instituto Balseiro en Bariloche y apoyó, junto a Carlos Mallmann y Enrique Oteiza (este último desde el Instituto Di Tella), la creación de la Fundación Bariloche, que acogió a numerosos académicos expulsados de la Universidad de Buenos Aires por la dictadura de Juan Carlos Onganía, en 1966.

La influencia de Sabato en estas actividades se debió mucho a la "rara mezcla de intuitivo y rigurosamente científico", y a su gran capacidad de liderazgo. Como él rememoraba de aquellos inicios de su gestión en la CNEA:

Nuestro laboratorio se inició sin metalurgistas, comenzando por el que habla, que no era metalurgista profesional sino de oficio (hecho a mano) que tenía entonces tres años y medio de trabajar en el laboratorio (que también había fundado) de una empresa local dedicada a la fabricación de cobre y aleaciones de cobre. Incorporé entonces a un ingeniero electromecánico (Martínez Vidal), a un ingeniero aeronáutico (Biloni), a varios ingenieros químicos (Mazza, Libonatti, Nelly Ambrosis, Kittl), a un ingeniero civil (Leyt), a tres licenciados en química (Coll, Carrea y Di Primio), a un estudiante de química (Carlos Aráoz), a otro ingeniero electromecánico (Oscar
Wortman), de los cuales ninguno sabía una letra de metalurgia (Sabato, 1972: 9).

En una entrevista en los comienzos de sus investigaciones sobre la Escuela PLACTED, en 1997, Martínez Vidal recalca:

La metalurgia no existía académicamente en el país. Para que dictasen materias de ese primer curso, invitamos a tres profesores europeos: Robert Cahn, de la universidad de Birmingham de Inglaterra, Pierre Lacombe, de la École de Mines de París, y Erich Gebhardt, del Max Planck Institut für Metallkunde de Stuttgart quienes dictaron algunas materias del Primer Curso de Postgrado en Metalurgia (Barros Medina, 1997: 8).

Sabato mismo había visitado centros de excelencia en metalurgia de varios países para asesorarse, donde fue recibido y respetado. Prueba de lo cual es que varias de esas instituciones, como las Universidades de Birmingham y de California, Io invitaron después como profesor visitante, Este intercambio facilitó, además, que pudiera enviar a formarse a los miembros de su Dirección de Metalurgia a centros altamente calificados de varios países.

Carlos Aráoz, uno de los primeros expertos del Departamento de Metalurgia, comentó en una de las entrevistas del Programa PLACTED (2011a: 13): "Sabato decía que había que hacer no la mejor metalurgia, pero sí había que hacerla al mejor nivel internacional. Y esa excelencia prendió, se mantuvo bastante. El elemento dinamizador era Sabato con sus contactos internacionales". Agregaba también lo siguiente:

En esa etapa, entre los que venían y los que salíamos a hacer estadías en el exterior, creo que conformó un equipo, una filosofía de trabajo que tenía buen nivel, que tenía conocimiento de que las cosas no eran imposibles. Una vez le dije a 
Sabato: "Pero, Jorge, acá en Inglaterra -cuando yo estaba allí- no es muy distinto a nosotros. Para eso los mandamos, me dijo, para que vean que no hay mucha diferencia". (PLACTED, 2011a: 13).

Para poder llevar a cabo esta enorme tarea, fue muy importante su capacidad de liderazgo. En una conversación con miembros del equipo del Programa PLACTED del MINCyT, en 2009, Enrique Oteiza nos decía sobre su personalidad:

En la discusión Sabato tenía la gran virtud de ser muy carismático y crear ciertos ámbitos de debate, para discutir el problema de la Tecnología con grupos chicos de industriales sensibles, que no estaban a la altura de lo que él sí sabía sobre esta cuestión, pero que se interesaban. Él sabía atraerlos, era un 'encantador de serpientes', pudiendo incluso convencer a algunos de ellos para que pusieran dinero....el grupo [de industriales] en síno era muy grande, lo que pasa es que logró impactar en algunas empresas metalúrgicas grandes, que fueron importantes para la construcción de partes del reactor nuclear [Atucha I]... Se armó incluso Astarsa [Astilleros Argentinos Río de La Plata S.A.], en su momento.

Desde este grupo se organizaron viajes a Francia que iban con Jorge Sabato de guía, se armaron para ver cómo era la industria en Francia, en particular la de proveedores de los reactores nucleares franceses. Tres o cuatro veces los llevó y los impactó, pues tenía mucho carisma. Hay empresarios todavía de esa época que me dicen que lo recuerdan a Jorge con respeto. Los ayudó de esa manera a conseguir el negocio, porque finalmente, parte de los reactores se hicieron acá y los que lo hicieron eran unas pocas industrias que tenían capacidad de hacerlo (PLACTED, 2009: 18, 20).
Se puede apreciar, pues, la gran influencia que tuvo Sabato tanto dentro de CNEA y en su propio equipo de trabajo, así como en el sector empresario metalúrgico argentino y en instituciones del exterior.

\section{Sabato y su influencia en el surgimiento deI PLACTED}

La capacidad de liderazgo de Sabato fue clave también para su rol en la creación y promoción del grupo de Pensamiento Latinoamericano en Ciencia Tecnología, Desarrollo y Dependencia. Consiguió aglutinar a un conjunto heterogéneo de actores. En particular a aquellos que habían participado en la generación de tecnologías propias en Ios más diversos sectores, como energía nuclear y aeronáutica en Argentina y Brasil, o siderurgia, petróleo, química y metalmecánica -en estos y otros países. Muchos de ellos a través del Programa Latinoamericano de Metalurgia impulsado por CNEA con apoyo de la OEA. En este organismo, a través del Programa Regional de Desarrollo Científico y Tecnológico, consiguió inculcar sus ideas, impulsadas por Carlos Martínez Vidal, Máximo Halty Carrère, Enrique Oteiza y Aldo Ferrer.

Es así como en 1975 publicó una compilación de artículos de los autores principales de este movimiento, en un libro, El pensamiento latinoamericano en la problemática ciencia-tecnología-desarrollo-dependencia, que el programa PLACTED del MINCyT reeditó en 2011 en colaboración con la Biblioteca Nacional (Sabato, 2011).

Una de las particularidades del PLACTED fue la conjunción entre pensamiento y acción. Al respecto, el propio Sabato señalaba:

Una de las características más singulares del proceso vivido en Latinoamérica alrededor de la problemática Ciencia-Tecnología-DesarroIlo-Dependencia fue la estrecha relación entre pensamiento y acción, es decir, entre la produc- 
ción de trabajos académicos referidos a distintos aspectos de la problemática y las medidas puestas en ejecución por instituciones nacionales y regionales para operar sobre la realidad en base a esos estudios (Sabato, 1972: 7).

Otros muchos conceptos que han marcado a este movimiento han tenido su origen e inspiración en el mismo Sabato y en su obra en la CNEA: el concepto de "tecnología como mercancía", la creación de fábricas de tecnología, que trató de promover en su breve paso como Presidente de Servicios Eléctricos del Gran Buenos Aires (SEG$\mathrm{BA}$, por entonces empresa de energía del conurbano bonaerense), la necesidad de un tratamiento integral de las políticas tecnológicas, articuladas con las políticas de desarrollo (o, como propuso Perón, con un Plan de Desarrollo) en lo que llamó "Régimen de Tecnología". Y finalmente, su propuesta tal vez más conocida, pero menos asimilada en su múltiple riqueza, el "Triángulo de Sabato".

\section{Prospectiva e innovación}

Sabato fue un pionero en ideas clave para el desarrollo tecnológico, como la prospectiva y el concepto de innovación. En el artículo sobre el Triángulo, conceptualiza por un lado el entramado ciencia-tecnología-innovación. Por otro lado expresa otras ideas novedosas, que lo constituyen en un auténtico visionario del mundo que vendría (Marí, 2012). En el punto cuatro de su artículo sobre el Triángulo expresa:

Es muy factible que los acontecimientos de las próximas décadas sean mucho más espectaculares y revolucionarios que los que han ocurrido en los últimos 20 años. En nuestros países es común pensar por el hecho de ser espectadores y no protagonistas, que estamos viviendo el momento culminante de la revolución científico-tecnológica. Ello no es cierto, como bien lo prueban los estudios prospectivos que de- muestran que son previsibles transformaciones cientificas mucho más profundas que las experimentadas hasta el presente (Sabato y Botana, 1968: 17).

Esta mención a los estudios prospectivos no es casual, sino que se fundaba en su experiencia al frente de un grupo de expertos latinoamericanos invitados por el Club de Roma en 1970 para que opinaran sobre los resultados de lo que sería luego el famoso informe "Límites al crecimiento" (Meadows, Meadows, Randers y Behrens III, 1972). Esta reunión derivó en el primer estudio latinoamericano de prospectiva, el "Modelo Mundial Latinoamericano" o "Modelo Bariloche", realizado entre 1971 y 1976 en la Fundación Bariloche, dirigido por Amílcar Herrera y con Hugo Scolnik a cargo del modelo de simulación numérica utilizado.

En el mismo artículo, después de proponer la figura del Triángulo, caracteriza a la infraestructura científico-tecnológica como un producto social (adelantándose a nivel mundial a estudios desarrollados con posterioridad desde la sociología de la ciencia). Introduce el concepto de innovación, al que define como "la incorporación del conocimiento -propio o ajeno- con el objeto de generar o modificar un proceso productivo" (Sabato y Botana, 1968: 19). Recordemos que hasta entonces las políticas de ciencia y tecnología no incluían el concepto de innovación, sino que se hablaba de productividad, cambio técnico, progreso tecnológico. Fue a través de la obra de Christopher Freeman, primero en sus trabajos para la UNESCO y luego desde su recién fundado Instituto de Estudios del Desarrollo, en la Science Policy Research Unit (SPRU) en la Universidad de Sussex, como empezó a difundirse este concepto, a raíz de su obra seminal de 1974, "The Economics of Industrial Innovation". Sabato se adelantó en seis años a estos análisis, en los mismos términos que Christopher Freeman utilizó para cimentar su teoría de la innovación y refutar el modelo lineal: 
Mientras sobre el tema de la investigación se conoce lo suficiente para saber lo que se debe $y$ lo que no se debe hacer para tener éxito, acerca del proceso de innovación, en cambio, es poco lo que se conoce: intervienen en él una cantidad de factores cuyo papel especifico e interrelación se desconocen; elementos de naturaleza tan dispar como la estructura económico-financiera de la sociedad y de las empresas, la movilidad social, la tradición, las características de los grupos dirigentes, el sistema de valores de la sociedad, las necesidades concretas en una situación determinada, los mecanismos de comercialización (Sabato y Botana, 1968: 20). ${ }^{1}$

No es extraña la coincidencia de Sabato y de la Escuela PLACTED con los desarrollos iniciados en Europa en la misma época, sobre todo si tenemos en cuenta que sus ideas, como las de los otros pensadores de la Escuela, están fundadas, como él mismo dijo, en su experiencia en el desarrollo y aplicación de tecnologías en la producción, y justamente en campos tan complejos como el nuclear, el aeronáutico y el petrolero.

Para concluir, cabe señalar otro elemento que Sabato resalta en el mismo artículo del Triángulo, un elemento que la moderna teoría evolucionista de la innovación con frecuencia olvidó: el rol del Estado. En efecto, esta visión moderna, en gran parte promovida por organismos internacionales como la OCDE (Organización para la Cooperación y el Desarrollo Económico) y el Banco Interamericano de Desarrollo, fue subsumida en los años '80 y '90 del siglo XX, a poco de su aparición, por el credo neoliberal, que minimizó el rol del Estado. Esto llevó, en el terreno práctico de las políticas, pero también de la investigación, a ubicar a la empresa (privada) como el locus cuasi único de la innova- ción y a la vinculación Academia-Industria como el eje de las nuevas políticas, olvidando el vértice del gobierno, bien señalado por la figura del triángulo. Se obviaba también la palabra "política" en favor de la de "gestión" (de la investigación y el desarrollo tecnológico).

Por otra parte, cuando Sabato describe "el sistema de relaciones" entre los vértices del triángulo, menciona como ejemplo paradigmático que "el proceso por el cual se estructura el sistema de relaciones en una sociedad" está claramente ilustrado por la experiencia de los Estados Unidos:

Durante la década de 1940 el gobierno [de aquel país] actúa sobre la infraestructura científico-tecnológica y la estructura productiva industrial en una escala mucho mayor de lo que había ocurrido anteriormente, convirtiéndose en el promotor más importante del proceso de innovación" (Sabato y Botana, 1968: 20).

Sabato era pues consciente del papel fundamental que el gobierno de los Estados Unidos había tenido, a partir de su demanda de producción bélica, en vincular y aplicar la ciencia y la tecnología al complejo militar-industrial, echando así las bases de las modernas políticas de ciencia y tecnología. Precisamente, "demanda" es el término que utiliza como fundamental en su sistema de relaciones. $Y$ como él mismo afirma después, la demanda del sector productivo es la que viene de las empresas, no importa que sean públicas o privadas.

Sabato era pues un convencido de la importancia del Estado, y también del rol de las empresas estatales. Fue designado por Aldo Ferrer, en 1970, como Presidente de SEGBA. Propuso, a partir de la misma, la creación de una Empresa Nacional de Investigación y Desarrollo Eléctrico (ENIDE), en

\footnotetext{
${ }^{1}$ Francisco Sagasti describe a Sabato como "uno de los pioneros y visionarios más importantes de la región en temas de ciencia, tecnología e innovación". Con la descripción de su figura del triángulo, afirma, rompe con el modelo lineal, e introduce en forma precursora el concepto de innovación, "adelantándose a la incorporación de la innovación como elemento articulador de la movilización del conocimiento para el desarrollo" (Sagasti, 2011: 102).
} 
base a su idea de "fábricas de tecnología". Renunció en 1971 a la Presidencia, probablemente en desacuerdo con el régimen militar del momento por su idea de la cogestión.

\section{Conclusiones}

Jorge Sabato falleció el 16 de noviembre de 1983, en el marco de la recuperación de la democracia, a unos días de la asunción del presidente Alfonsín. Víctima de un cáncer, estuvo activo hasta último momento, ya que en una suerte de autoexilio había estado investigando sobre uno de sus temas preferidos: la comercialización de tecnología, en una Universidad canadiense. Además combatió a lo que él llamaba "el partido militar" desde diversos ámbitos, en particular desde la revista Humor. Poco antes de morir se lo mencionaba como candidato a ocupar el cargo de primer Secretario de Ciencia y Tecnología del gobierno recién elegido.

Acerca de sus convicciones políticas, vale rescatar lo expresado en una entrevista que le realizaran en 1971, reproducida por Federico García Blaya (2020).

En materia política soy de extracción y convicciones democráticas... partidario de todo lo que contribuya a la personalidad del pueblo y de la nación... en el sentido de que se pueden hacer cosas por la nación, en la nación y para la nación... entonces soy nacionalista, ni tropical ni extemporáneo... no sé qué rótulo ponerle... una mezcla rara de Museta y de Mimín ${ }^{2}$ de peronismo y otras cosas que quiera ponerle, sin ser peronista...

Su visión se radicalizó en sus últimos meses. Personas cercanas a él, como Sara Rietti (Programa
PLACTED, 2011b: 2) afirmaba que en una conversación personal le dijo: "Varsavsky tenía razón", refiriéndose a la falacia de la neutralidad de la ciencia, y a la necesidad de un proyecto nacional en el que la ciencia y la tecnología estuvieran abocadas a resolver la necesidades del país, como siempre lo había propugnado.

\section{Bibliografía}

Barrios Medina, A. (1997). La escuela latinoamericana de pensamiento en ciencia, tecnología y desarrollo (CTD) a través del Dr. Ing. Carlos Martínez Vidal (mimeo).

Ciapuscio, H. (comp.) (1994). Repensando la política tecnológica, Homenaje a Jorge A. Sabato. Buenos Aires: Nueva Visión.

Galante, O., Benso, O., Carnota, R., Marí, M. y Vasen, F. (2009). "La Escuela Latinoamericana de Pensamiento en Ciencia, Tecnología y Desarrollo", Congreso de la Asociación Latino-Iberoamericana de Gestión Tecnológica (ALTEC), México.

García Blaya, F. (4 de junio de 2020). El Laberinto. Columna de tango. Radio Rebelde 740. Recuperado de: https://ar.radiocut.fm/audiocut/en-laberinto-4-6-2020-columna-tango-federico-garcia-blaya/ [fecha de consulta: 2-9-2020]

Marí, M. (2012). Historia del concepto de Innovación. Ponencia presentada en las IX Jornadas Latinoamericanas de Estudios Sociales de la Ciencia y la Tecnología, ESOCITE, México.

Meadows, D. H., Meadows, Dennis L., Randers, J. y Behrens III, W. W. (1972). Limits to Growth. New York: Signet Books.

Martínez Vidal, C. y Marí, M. (2002). La Escuela La-

\footnotetext{
2 Museta y Mimí son dos personajes del tango Griseta (Sabato era muy aficionado al tango), que representan a dos mujeres de extracción y características muy disímiles. Con esta referencia, podríamos decir que Sabato se identifica con las banderas del peronismo, sin formar parte del partido. Algo así como en esos mismos tiempos los cristianos postconciliares y el Movimiento de Curas del Tercer Mundo llamaban a sus hermanos marxistas como "cristianos anónimos".
} 
tinoamericana de Pensamiento en Ciencia, Tecnología y Desarrollo. Revista REDES, № 19.

Programa PLACTED/MINCYT (2009). "Entrevista a Enrique Oteiza".

Programa PLACTED/MINCYT (2011a). "Entrevista a Carlos Aráoz, integrante del equipo de Metalurgia de la CNEA de J. Sabato", Buenos Aires.

Programa PLACTED/MINCYT (2011b). “Entrevista a Sara Rietti", Buenos Aires.

Sabato, J. y Botana, N. (1968). La ciencia y la tecnología en el desarrollo futuro de América Latina. Revista de la Integración, INTAL, Buenos Aires, Año 1, Nro. 3, pp. 15-36.

Sabato, J. A. (1972). Quince años de metalurgia en la Comisión Nacional de Energía Atómica. Ciencia Nueva, No. 15, pp. 7-15.
Sabato, J. A. (comp.) (2011). El pensamiento latinoamericano en la problemática ciencia-tecnología-desarrollo-dependencia. Buenos Aires: Ediciones Biblioteca Nacional.

Sagasti, F. (2011). Ciencia, Tecnología e Innovación. Politicas para América Latina. Lima: Siglo XXI. 